\title{
STAR Highlights
}

\author{
Hiroshi Masui for the STAR collaboration \\ Lawrence Berkeley National Laboratory, Nuclear Science Division, 1 Cyclotron Road, \\ Berkeley, CA 94720 \\ E-mail: HMasui@lbl.gov
}

\begin{abstract}
We report selected results from STAR collaboration at RHIC, focusing on jet-hadron and jet-like correlations, quarkonium suppression and collectivity, dielectron spectrum in both $\mathrm{p}+\mathrm{p}$ and $\mathrm{Au}+\mathrm{Au}$, and higher moments of net-protons as well as azimuthal anisotropy from RHIC Beam Energy Scan program.
\end{abstract}

\section{Introduction}

The main goals at the STAR experiment for ultra-relativistic heavy ion collisions is to study the structure of QCD phase diagram. In order to achieve this question, the STAR experiment has two main programs in heavy ion collisions; study the medium properties and eventually identify the equation of state of partonic matter, and search for the QCD critical point through RHIC Beam Energy Scan (BES).

This article is organized as follows. In Section 2, recent results on jet-hadron and jetlike correlations are presented, which can be used to understand underlying mechanisms of jet-quenching and hadron productions at higher $p_{T}$. The suppression and collectivity for heavy flavors are discussed through the measurement of the transverse momentum $p_{T}$ spectra, nuclear modification factor $R_{A A}$ and azimuthal anisotropy $v_{2}$ in Section 3 , In Section 4 , the first STAR measurement of di-electron spectrum in both $\mathrm{p}+\mathrm{p}$ and $\mathrm{Au}+\mathrm{Au}$ are presented. Section 5 presents selected results from BES about higher moments of net-protons and second harmonic of azimuthal anisotropy $v_{2}$ for identified hadrons

\section{Jet-hadron and jet-like correlations}

Measurements of $R_{A A}$ as well as di-hadron azimuthal correlations for high $p_{T}$ inclusive charged hadrons have indicated large partonic energy loss in the medium at RHIC [1, 2]. Jet-hadron correlation with full jet reconstructions can provide direct access to the energy of partons in order to understand the underlying mechanisms how partons lose energy in the medium. Figure 1 (left) shows the away side $(\Delta \phi \sim \pi)$ energy 

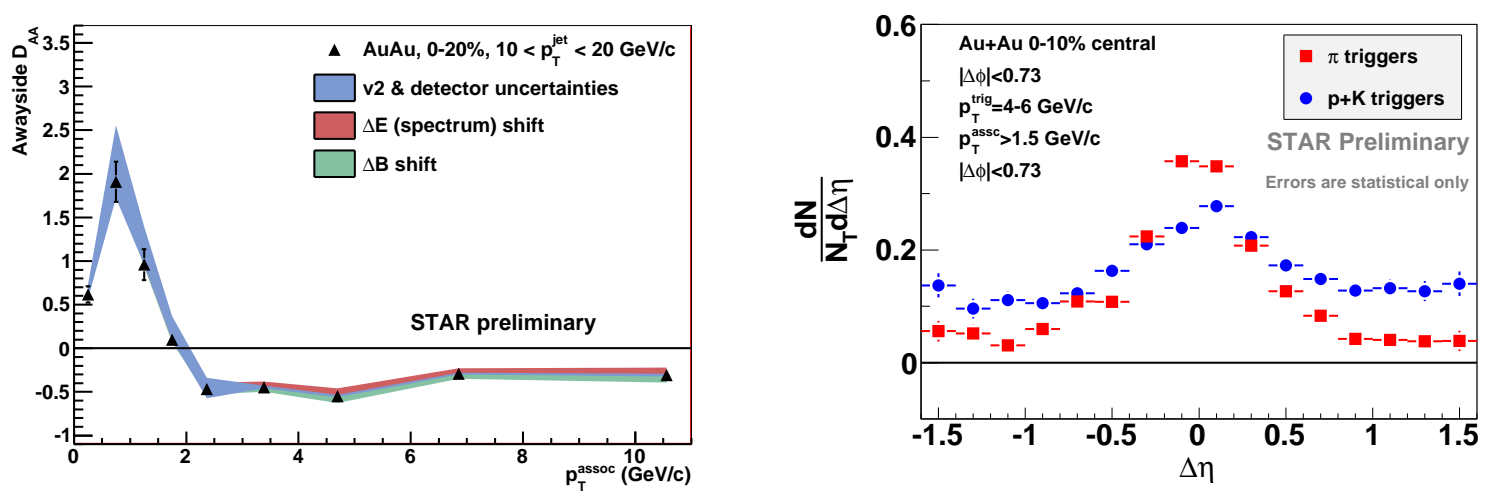

Figure 1. (Color online) (Right) Away side energy balance $D_{A A}$ as a function of associated hadron $p_{T}\left(p_{T}^{a s s o c}\right)$ in $0-20 \% \mathrm{Au}+\mathrm{Au}$ collisions at $\sqrt{s_{N N}}=200 \mathrm{GeV}$, where the bands represent systematic uncertainties arising from several different sources quoted in the figure. (Left) $\Delta \eta$ distributions for $\pi$ (squares) and $p+K$ (circles) triggered hadron correlation in central $0-10 \% \mathrm{Au}+\mathrm{Au}$ collisions at $\sqrt{s_{N N}}=200 \mathrm{GeV}$. Only statistical errors are shown.

balance $D_{A A}$ as a function of associated hadron $p_{T}^{a s s o c}$ in $0-20 \% \mathrm{Au}+\mathrm{Au}$ collisions at $\sqrt{s_{N N}}=200 \mathrm{GeV}[3]$. The $D_{A A}$ is defined by

$$
D_{A A}\left(p_{T}^{a s s o c}\right)=Y_{A A}\left(p_{T}^{a s s o c}\right) \cdot p_{T, A A}^{a s s o c}-Y_{p p}\left(p_{T}^{a s s o c}\right) \cdot p_{T, p p}^{a s s o c},
$$

where $Y\left(p_{T}^{a s s o c}\right)$ denotes the away side jet yields. In central $0-20 \%$, the away side jets are significantly enhanced at low $p_{T}^{a s s o c}$, and suppressed at high $p_{T}^{a s s o c}$. The integrated energy balance for the away side jets $\Delta B=1.6_{-0.4-0.4}^{+1.6+0.5} \mathrm{GeV} / c$ reveals a large part of high $p_{T}^{a s s o c}$ suppression is balanced by low $p_{T}^{a s s o c}$ enhancement. This result together with the observation of significant broadening of awayside jets in $\mathrm{Au}+\mathrm{Au}$ compared to $\mathrm{p}+\mathrm{p}$ seems to be consistent with the radiative energy loss picture.

The $p / \pi$ ratio at intermediate $p_{T}\left(2<p_{T}<6 \mathrm{GeV} / c\right)$ have been qualitatively described by recombination models in the most central $\mathrm{Au}+\mathrm{Au}$ collisions [2], which indicates that coalescence or recombination process would play an important role for hadron productions at intermediate $p_{T}$. More differential study by utilizing the dihadron correlation with identified particles would provide further insights into the hadron production mechanisms at intermediate $p_{T}$. Figure 1 (right) shows the $\Delta \eta$ distributions for identified $\pi$ and $p+K$ in $4<p_{T}^{\text {trig }}<6 \mathrm{GeV} / c$ associated with inclusive charged hadrons in $1.5<p_{T}^{\text {assoc }}<p_{T}^{\text {trig }} \mathrm{GeV} / c$ in the most central $0-10 \% \mathrm{Au}+\mathrm{Au}$ collisions [4], where $\eta$ denotes the pseudorapidity. The $\pi$ and $p+K$ are identified by their specific energy loss in the Time Projectioin Chamber (TPC). The shoulder structures in the large $\Delta \eta$ (ridge) is higher for $p+K$ trigger than that for $\pi$ trigger. The near side peak is higher for $\pi$ trigger compared to that for $p+K$ trigger, which is similar in both $\mathrm{d}+\mathrm{Au}$ and $\mathrm{Au}+\mathrm{Au}$ collisions. The similarity of near side peak in $\mathrm{d}+\mathrm{Au}$ and $\mathrm{Au}+\mathrm{Au}$ collisions pose a challenge to the naive recombination picture, where the dilution of near side peak with thermalized partons would be expected if the recombination is the dominant mechanism of hadron productions at intermediate $p_{T}$. 


\section{Heavy flavors}

The suppression of $J / \psi$ due to color screening of $c \bar{c}$ pairs is expected to be one of the unambiguous signature of QGP formation [5]. Measurements of nuclear modification factor $R_{A A}$ for $J / \psi$ in $\sqrt{s_{N N}}=200 \mathrm{GeV} \mathrm{Au}+\mathrm{Au}$ collisions at RHIC show the similar suppression pattern as at SPS as a function of centrality [6], while the $R_{A A}$ for high $p_{T}\left(p_{T}>5 \mathrm{GeV} / c\right) \mathrm{J} / \psi$ is consistent with no suppression in $\mathrm{Cu}+\mathrm{Cu}$ collisions [7]. It has been reported that the suppression of $\Upsilon$ at RHIC could provide large sensitivity to color screening in the QGP because contributions from regeneration [8] and absorption by hadronic co-movers are negligible [9]. The quarkonium suppression would be one of the probes to study the properties of the QGP as well as to understand their production mechanisms.
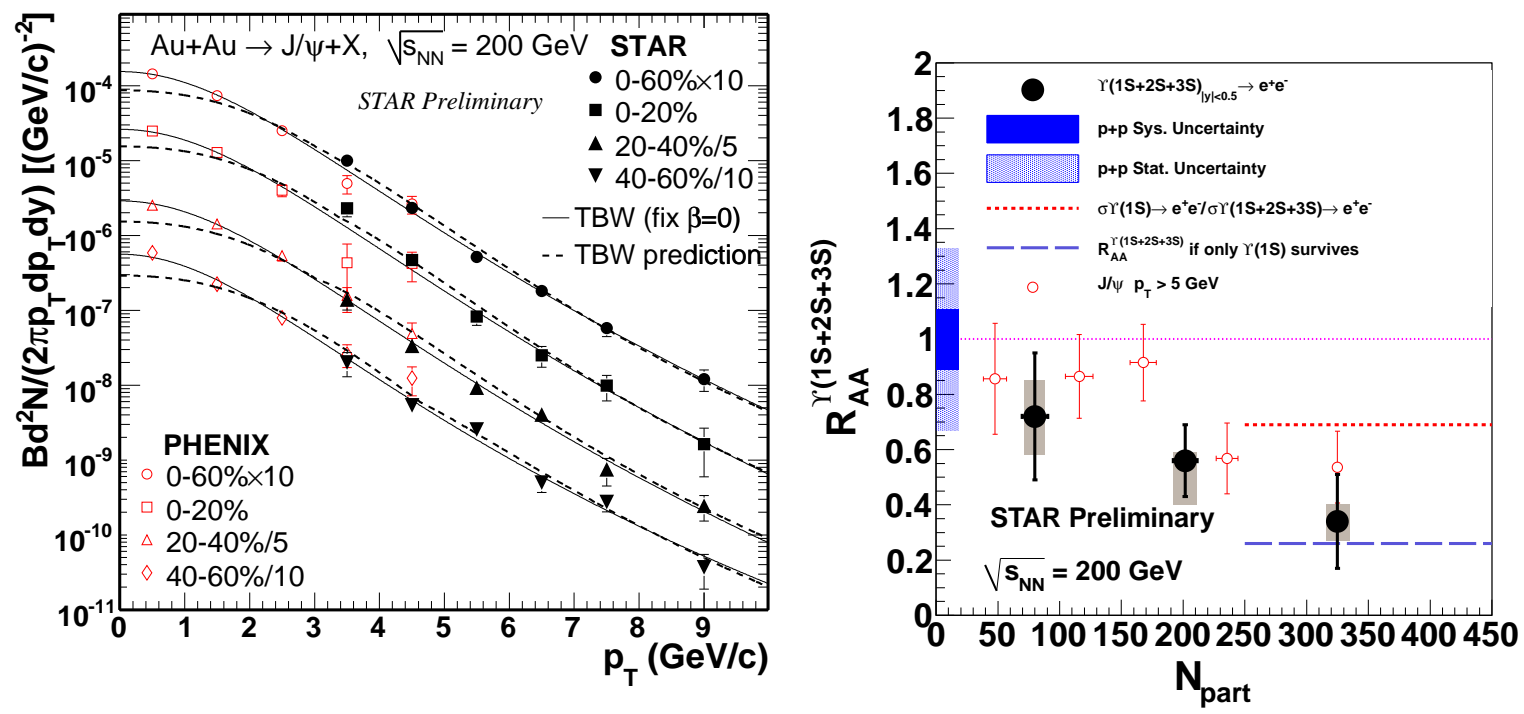

Figure 2. (Color online) (Right) Invariant yields of $J / \psi$ as a function of $p_{T}$ for several different centrality bins shown by solid symbols in $\mathrm{Au}+\mathrm{Au}$ collisions at $\sqrt{s_{N N}}=200 \mathrm{GeV}$. Open symbols are published PHENIX results for comparison [6]. Lines are calculations from Tsallis-Blast-Wave model [10. (Left) $\Upsilon(1 \mathrm{~S}+2 \mathrm{~S}+3 \mathrm{~S}) R_{A A}$ as a function of $N_{\text {part }}$ compared to the $J / \psi R_{A A}$ for $p_{T}>5 \mathrm{GeV} / c$ in $\mathrm{Au}+\mathrm{Au}$ collisions at $\sqrt{s_{N N}}=200 \mathrm{GeV}$. The shaded bands at $N_{\text {part }}=0$ represent statistical and systematic uncertainties in $\mathrm{p}+\mathrm{p}$ measurements.

Figure 2 (left) shows the invariant yields of $J / \psi$ as a function of $p_{T}$ in $\mathrm{Au}+\mathrm{Au}$ collisions at $\sqrt{s_{N N}}=200 \mathrm{GeV}$ for several different centrality bins. High statistics data in year 2010 allows us to extend the $p_{T}$ reach of $J / \psi$ spectra out to $10 \mathrm{GeV} / c$ [11]. Figure2 (right) shows the $R_{A A}$ for high $p_{T} J / \psi\left(p_{T}>5 \mathrm{GeV} / c\right)$ and $\Upsilon(1 \mathrm{~S}+2 \mathrm{~S}+3 \mathrm{~S})$ as a function of number of participants $N_{\text {part }}$ in $\mathrm{Au}+\mathrm{Au}$ collisions at $\sqrt{s_{N N}}=200 \mathrm{GeV}$. The high $p_{T} J / \psi$ shows suppression in central collisions, while the magnitude of $R_{A A}$ is higher in higher $p_{T}$ compared to the measurements at low $p_{T}$ from [6]. The $\Upsilon(1 \mathrm{~S}+2 \mathrm{~S}+3 \mathrm{~S}) R_{A A}$ shows similar suppression pattern as high $p_{T} J / \psi$. The $R_{A A}$ in the most central $0-10 \%$ indicates the suppression of $\Upsilon$, which is more than $3 \sigma$ away from unity, with systematic 
uncertainties in $\mathrm{p}+\mathrm{p}[12]$.

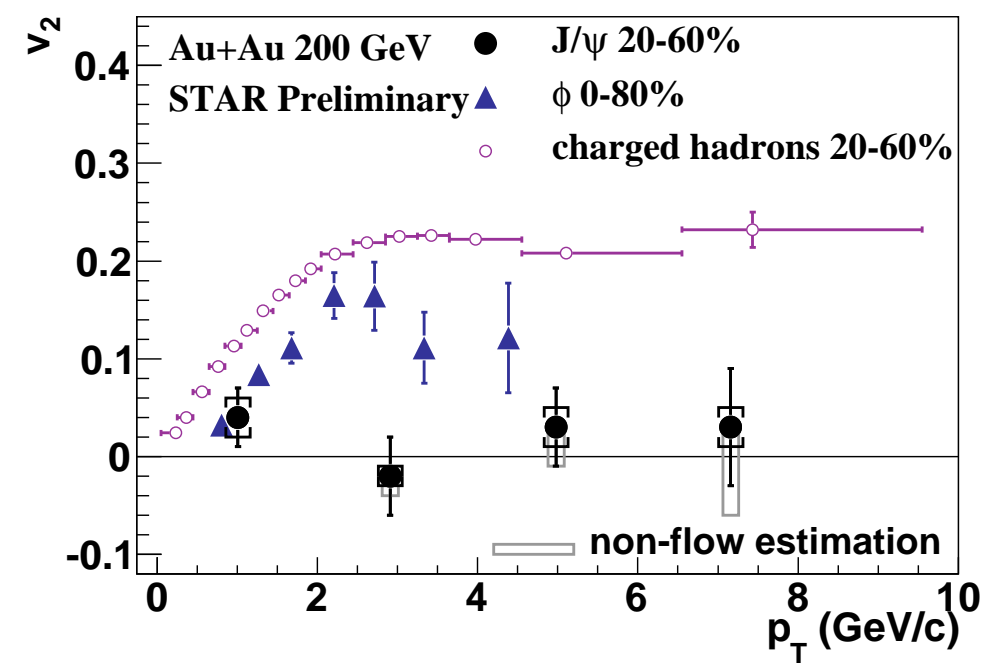

Figure 3. (Color online) $J / \psi v_{2}$ (solid circles) as a functioin of $p_{T}$ in mid-central $20-60 \% \mathrm{Au}+\mathrm{Au}$ collisions at $\sqrt{s_{N N}}=200 \mathrm{GeV}$, where open boxes show systematic uncertainties from non-flow effects, and brackets show those from other sources. Open circles show result of inclusive charged hadrons in 20-60\% 13] and solid triangles are the $\phi$ meson $v_{2}$ in $0-80 \%$ [14] for comparison.

It has been reported at RHIC that azimuthal anisotropy $v_{2}$ have shown universal scaling among light flavor mesons and baryons in intermediate $p_{T}$ range suggested by parton coalescence and recombination models [14, 15, 16, 17]. The results are strong indication of partonic degrees of freedom in the early stage of heavy ion collisions. Measurements of charm quark collectivity could play a potential role to answer the thermalization of light flavors as well as to shed more lights on the production mechanisms of charm quarks in the medium. Figure 3 shows new measurements of $J / \psi v_{2}$ as a function of $p_{T}$ in 20-60\% Au + Au collisions at $\sqrt{s_{N N}}=200 \mathrm{GeV}$, up to $p_{T}=8 \mathrm{GeV} / c$ [11]. Systematic errors from non-flow effects are evaluated with measurements of preliminary $J / \psi$-hadron correlation in $\mathrm{p}+\mathrm{p}$ collisions by assuming that the non-flow effects are the same in $\mathrm{p}+\mathrm{p}$ and $\mathrm{Au}+\mathrm{Au}$ collisions. The $J / \psi v_{2}$ appears to be consistent with 0 within systematic uncertainties for $p_{T}>2 \mathrm{GeV} / c$, which seems to disfavor the coalescence scenario to form $J / \psi$ with thermalized charm quarks.

\section{Electromagnetic probes}

Di-lepton productions are of particular interest among the observables to explore the early stage of heavy ion collisions. They directly probe the evolution of the medium and unaffected by final-state interactions in contrast to hadrons.

Figure 4 presents the recent measurements of STAR di-electron mass spectrum in both $\mathrm{p}+\mathrm{p}$ (left) and $\mathrm{Au}+\mathrm{Au}$ (right) collisions at $\sqrt{s_{N N}}=200 \mathrm{GeV}$ after combinatorial 

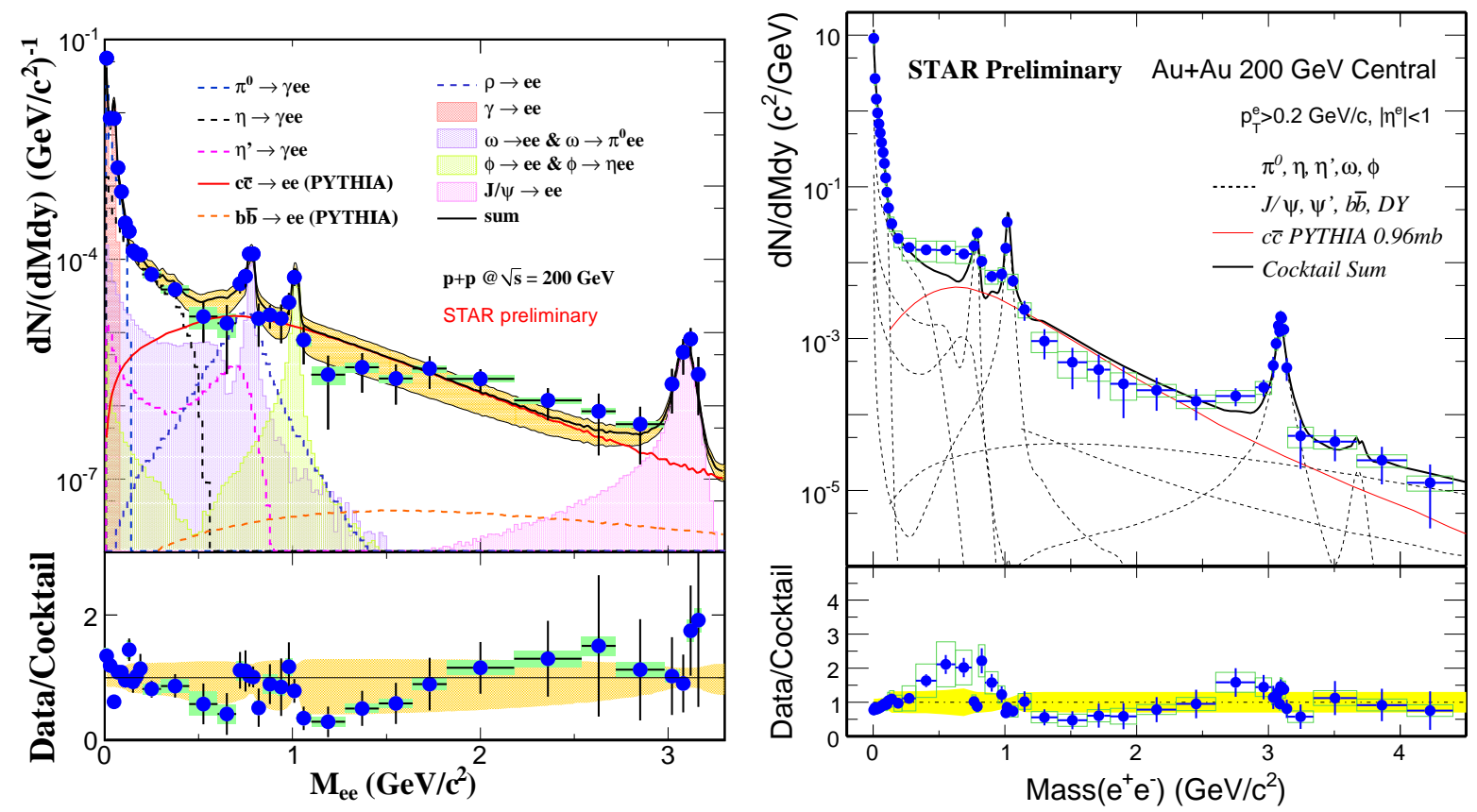

Figure 4. (Color online) Di-electron mass spectrum in $\mathrm{p}+\mathrm{p}$ collisions at $\sqrt{s}=200 \mathrm{GeV}$ (left) and in $\mathrm{Au}+\mathrm{Au}$ collisions at $\sqrt{s_{N N}}=200 \mathrm{GeV}$ (right) after combinatorial background subtraction. Solid circles show the data, the bars denote the statistical uncertainties and boxes around data points show systematic uncertainties. The dashed lines represent the individual hadronic source used in the cocktail simulations. Bottom panels show the ratio of data to cocktail simulation, where shaded bands around unity and the boxes around data points show the systematic uncertainties on cocktail and data, respectively.

background subtraction [18]. The result in $\mathrm{p}+\mathrm{p}$ collicions is consistent with the know hadronic sources by cocktail simulation within systematic uncertainties, which provides the baseline for the measurements in $\mathrm{Au}+\mathrm{Au}$ collisions. The result in central 0-5\% $\mathrm{Au}+\mathrm{Au}$ collisions is also compared to the hadronic cocktail simulation as shown in the right panel. The data in $M\left(e^{+} e^{-}\right)<1 \mathrm{GeV} / c^{2}$ appears to be enhanced by a factor of $1.72 \pm 0.10$ (stat) \pm 0.50 (sys) compared to the cocktail simulation without $\rho^{0}$ contribution in the cocktail. Further study will be done to study the possible modification of $\rho^{0}$ in the low mass region and charm contributions in the intermediate mass region.

\section{Beam Energy Scan}

The goal of RHIC Beam Energy Scan is to search for the QCD critical point, onset of signature of QGP, and softening of equation of state. It has been started since year 2010, and STAR experiment collected the data at $\sqrt{s_{N N}}=7.7,11.5$ and $39 \mathrm{GeV}$ in 2010, and $19.6 \mathrm{GeV}$ in 2011.

Higher moments of fluctuations for conserved quantities, such as net-baryons, netcharge and so on, are expected to be more sensitive to the correlation length of the system, and thus to be a powerful tool to search for the critical point [19, 20]. Figure 5 

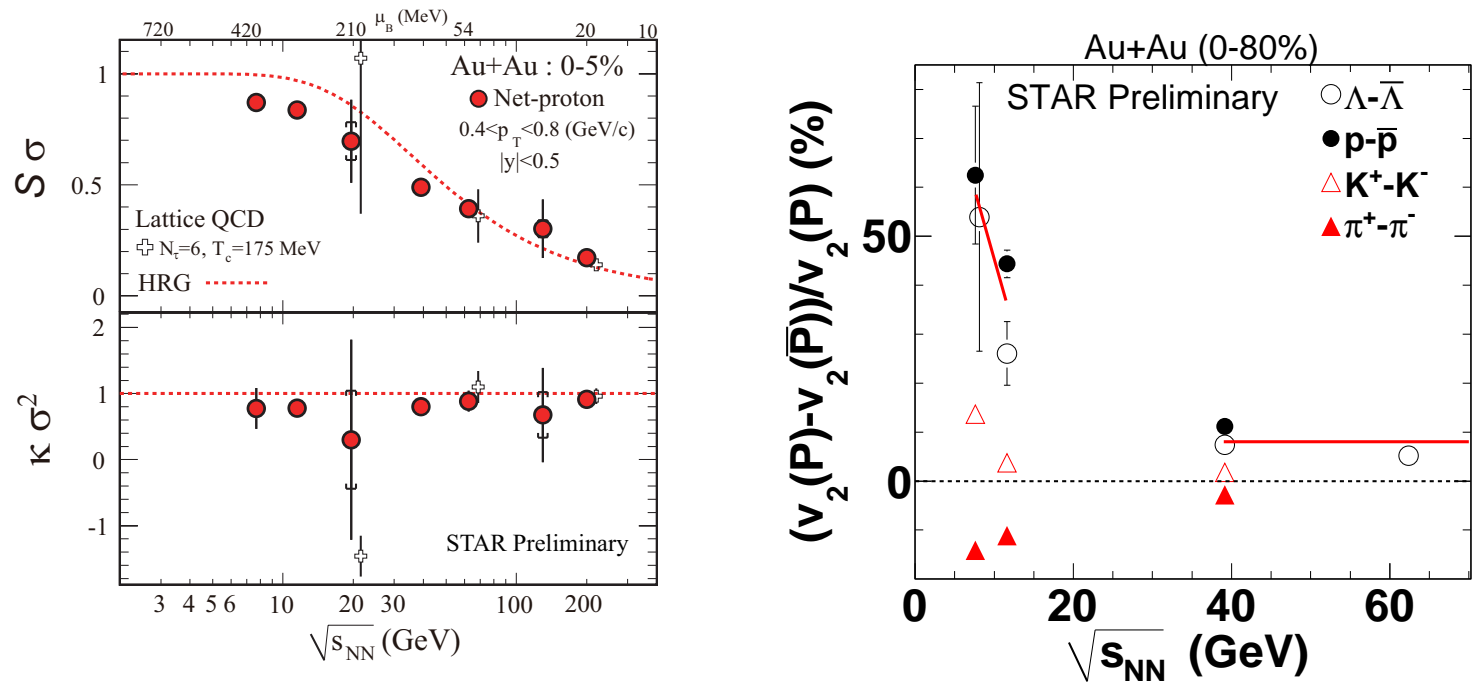

Figure 5. (Color online) (Left) Product of higher moments $S \sigma$ (top) and $\kappa \sigma^{2}$ (bottom) for net-protons as a function of $\sqrt{s_{N N}}$ in the most central $\mathrm{Au}+\mathrm{Au}$ collisions. Dashed lines show the calculations from Hadron Resonance Gas model [22] and open crosses show the Lattice QCD calculations [23]. (Right) Difference of $v_{2}$ between particles and anti-particles normalized by particle $v_{2}$ as a function of $\sqrt{s_{N N}}$ in $0-80 \%$ $\mathrm{Au}+\mathrm{Au}$ collisions for $\Lambda$ (open circles), $p$ (solid circles), $K$ (open triangles) and $\pi$ (solid triangles). The data points for $\Lambda$ at $\sqrt{s_{N N}}=62.4 \mathrm{GeV}$ are taken from [25]. Solid lines just guide eyes.

(left) shows the excitation function of the product of net-proton higher moments $S \sigma$ and $\kappa \sigma^{2}$ in the most central $\mathrm{Au}+\mathrm{Au}$ collisions, where $\sigma^{2}, S$ and $\kappa$ denote the variance, skewness and kurtosis, respectively [21. The calculations from Hadron Resonance Gas (HRG) [22] model as well as Lattice QCD [23] are also shown for comparison. The data at $\sqrt{s_{N N}}=62.4-200 \mathrm{GeV}$ are consistent with both HRG and Lattice QCD results, while the results at $\sqrt{s_{N N}}=7.7-39 \mathrm{GeV}$ show deviation from the HRG model. The data at $\sqrt{s_{N N}}=19.6 \mathrm{GeV}$ show the largest deviation with huge statistical uncertainty, which will be improved by using the new $19.6 \mathrm{GeV}$ data with much higher statistics.

As discussed earlier, the number of quark scaling of $v_{2}$ could be the strong indication of partonic degrees of freedom at $\sqrt{s_{N N}}=200 \mathrm{GeV}$. The observed scaling can be used as a turn-off signature if the hadronic stage becomes dominant in lower energies at BES. Figure 5 (right) shows the excitation function of the difference of $v_{2}$ between particles and anti-particles normalized by particle $v_{2}$ in $0-80 \% \mathrm{Au}+\mathrm{Au}$ collisions at $\sqrt{s_{N N}}=7.7$ $62.4 \mathrm{GeV}$ [24]. The results at $62.4 \mathrm{GeV}$ has shown about $5 \%$ difference of $v_{2}$ between $\Lambda$ and $\bar{\Lambda}$ [25]. At $\sqrt{s_{N N}}=7.7$ and $11.5 \mathrm{GeV}$, the difference of $v_{2}$ is significantly larger $\sim$ $50 \%$ for baryons than those at higher energies. The observed difference is similar for $p$ and $\Lambda$. There are $5-10 \%$ difference on meson $v_{2}$ as well at $\sqrt{s_{N N}}=7.7$ and $11.5 \mathrm{GeV}$. 


\section{Summary}

We report recent results from STAR experiment at RHIC. The away side jets from jethadron correlation in $\mathrm{Au}+\mathrm{Au}$ collisions show significant modifications as compared to that in $\mathrm{p}+\mathrm{p}$ collisions. The results seem to be consistent with the radiative energy loss picture. The near side peak obtained by di-hadron correlation with identified $\pi$ trigger is higher than that for $p+K$ trigger, which is similar in both $\mathrm{d}+\mathrm{Au}$ and $\mathrm{Au}+\mathrm{Au}$ collisioins. This result appears to disfavor the expectations from naive coalescence of thermal partons. The high $p_{T} J / \psi$ shows stronger suppression in more central collisions, while the magnitude of suppression is less compared to the results in lower $p_{T}$. The suppression of $\Upsilon$ is observed at $3 \sigma$ level including $\mathrm{p}+\mathrm{p}$ uncertainties at most central $0-10 \%$ collisions. The $J / \psi v_{2}$ appears to be consistent with 0 in $2<p_{T}<8 \mathrm{GeV} / c$, which disfavors the scenario of naive coalescence for thermal charm quarks. The STAR new measurement of di-electron mass spectrum in $\mathrm{Au}+\mathrm{Au}$ collisions at $\sqrt{s_{N N}}=200 \mathrm{GeV}$ is presented compared to that in $\mathrm{p}+\mathrm{p}$ collisions. The result in $\mathrm{p}+\mathrm{p}$ collisions is consistent with hadronic cocktail simulation, which provides the baseline for the measurements in $\mathrm{Au}+\mathrm{Au}$ collisions. The enhancement of di-electron spectrum is observed below $M\left(e^{+} e^{-}\right)<1 \mathrm{GeV} / c^{2}$ in $\mathrm{Au}+\mathrm{Au}$ collisions. Further investigation will be carried out to understand the possible modification of vector mesons as well as charm contributions. Excitation function of higher moments of net-protons are reported. The results are in good agreement with HRG model at higher energies, while they seems to deviate from the HRG model in $\sqrt{s_{N N}}=7.7-39 \mathrm{GeV}$. Significant difference between baryon and antibaryon $v_{2}$ is observed for $p$ and $\Lambda$ in $\sqrt{s_{N N}}=7.7$ and $11.5 \mathrm{GeV}$. Further studies will be performed with 19.6 and $27 \mathrm{GeV}$ data in order to search for the QCD critical point and the onset of QGP.

\section{References}

[1] J. Adams et al. [STAR Collaboration], Phys. Rev. Lett. 91, 172302 (2003)

[2] C. Adler et al. [STAR Collaboration], Phys. Rev. Lett. 90, 082302 (2003)

[3] A. Ohlson [STAR Collaboration], these proceedings

[4] K. Kauder [STAR Collaboration], these proceedings

[5] T. Matsui and H. Satz, Phys. Lett. B 178, 416 (1986).

[6] A. Adare et al. [PHENIX Collaboration], Phys. Rev. Lett. 98, 232301 (2007)

[7] B. I. Abelev et al. [STAR Collaboration], Phys. Rev. C 80, 041902 (2009)

[8] L. Grandchamp, S. Lumpkins, D. Sun, H. van Hees and R. Rapp, Phys. Rev. C 73, 064906 (2006)

[9] Z. w. Lin and C. M. Ko, Phys. Lett. B 503, 104 (2001)

[10] Z. Tang et al., arXiv:1101.1912 [nucl-ex].

[11] Z. Tang [STAR Collaboration], these proceedings

[12] R. Reed [STAR Collaboration], these proceedings

[13] J. Adams et al. [STAR Collaboration], Phys. Rev. Lett. 93, 252301 (2004)

[14] B. I. Abelev et al. [STAR Collaboration], Phys. Rev. Lett. 99, 112301 (2007)

[15] D. Molnar and S. A. Voloshin, Phys. Rev. Lett. 91, 092301 (2003)

[16] V. Greco, C. M. Ko and P. Levai, Phys. Rev. C 68, 034904 (2003)

[17] R. J. Fries, B. Muller, C. Nonaka and S. A. Bass, Phys. Rev. C 68, 044902 (2003)

[18] J. Zhao [STAR Collaboration], these proceedings 
[19] M. A. Stephanov, Phys. Rev. Lett. 102, 032301 (2009)

[20] M. M. Aggarwal et al. [STAR Collaboration], Phys. Rev. Lett. 105, 022302 (2010)

[21] T. Tarnowsky [STAR Collaboration], these proceedings

[22] F. Karsch and K. Redlich, Phys. Lett. B 695, 136 (2011)

[23] S. Gupta, X. Luo, B. Mohanty, H. G. Ritter and N. Xu, Science 332, 1525 (2011)

[24] A. Schmah [STAR Collaboration], these proceedings

[25] B. I. Abelev et al. [the STAR Collaboration], Phys. Rev. C 75, 054906 (2007) 